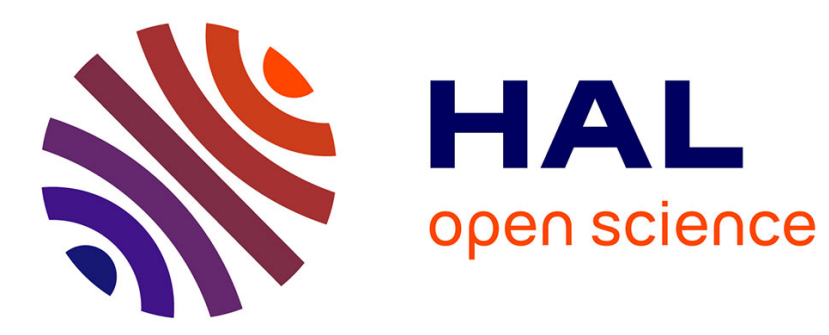

\title{
Generation of high quality tunable Bessel beams using a liquid-immersion axicon
}

\author{
Selcuk Akturk, Cord L. Arnold, Bernard Prade, André Mysyrowicz
}

\section{To cite this version:}

Selcuk Akturk, Cord L. Arnold, Bernard Prade, André Mysyrowicz. Generation of high quality tunable Bessel beams using a liquid-immersion axicon. Optics Communications, 2009, 282 (16), pp.3206. 10.1016/j.optcom.2009.05.026 . hal-00458426

\section{HAL Id: hal-00458426 \\ https://hal-polytechnique.archives-ouvertes.fr/hal-00458426}

Submitted on 5 Mar 2010

HAL is a multi-disciplinary open access archive for the deposit and dissemination of scientific research documents, whether they are published or not. The documents may come from teaching and research institutions in France or abroad, or from public or private research centers.
L'archive ouverte pluridisciplinaire HAL, est destinée au dépôt et à la diffusion de documents scientifiques de niveau recherche, publiés ou non, émanant des établissements d'enseignement et de recherche français ou étrangers, des laboratoires publics ou privés. 


\title{
Generation of high quality tunable Bessel beams using a liquid-immersion axicon
}

\author{
Selcuk Akturk," Cord L. Arnold, Bernard Prade, and Andre Mysyrowicz \\ Laboratoire d'Optique Appliquée, École Nationale Supérieure des Techniques Avancées-École \\ Polytechnique, CNRS UMR 7639 F-91761 Palaiseau Cedex, France \\ "Corresponding author: selcuk.akturk@ensta.fr
}

\begin{abstract}
By immersing a conventional glass axicon in index-matching liquid, we generated high quality, tunable, quasi non-diffracting Bessel beams. The aberrations resulting from the roundness of the axicon tip are minimized when a large base angle is used in liquidimmersion. This configuration also allows coarse and fine tunability through changing the liquid and adjusting the temperature, respectively. Our experimental results match very well with calculated intensity profiles. We succeeded to generate two-meter long plasma channels in air by focusing femtosecond laser pulses with the liquid immersion axicon.
\end{abstract}

\section{Introduction}

The virtues of non-diffracting beams are appealing particularly when high laser intensities are needed over prolonged distances. These beams are proper solutions to the wave equation when the transverse field profile is in the form of zeroth-order Bessel function [1]. While the ideal Bessel beams carry infinite energy, hence are unpractical, approximate Bessel-like profiles can be generated, retaining high intensities over distances much longer compared to Gaussian beams of similar spot sizes. This behavior proved fruitful for many applications ranging from optical coherence tomography [2] and multi-photon imaging [3,4] to manipulation and sorting of micro objects like biological cells [5-7] and generation of nonlinear optical interactions (including plasma formation) in solids [8-10] liquids [4, 11-13] and gases [14-17]. Several approaches have been shown to reasonably approximate the required beam profile, including double-slit with focusing lens [18], circular periodic gratings [19], tunable acoustic gradients [20] and axicon focusing [21]. Axicon focusing is superior to other methods in terms of energy throughput. However, a conventional axicon generates Bessel-like beam with certain spatial frequency determined by the cone angle; hence, the element is not tunable. Moreover, due to manufacturing constraints, the tip of the axicon deviates from the ideal cone shape and becomes rather round, causing significant aberrations in the intensity profile [22-24] (introducing modulations in the on-axis intensity). These oscillations result from the interference of parts of the incoming beam propagating through the round, lens-like axicon tip and the conical surface surrounding the tip [22]. Axicons with smaller base angles (longer Bessel zones) are more prone to these aberrations. 
Due to these aberrations and manufacturing difficulties, base angles smaller than $\sim 0.5^{\circ}$ are unpractical.

In this work we present an efficient solution to these limitations. We introduce liquidimmersion axicon, which not only allows tunability from large up to very small cone angles, but also eliminates the aberrations resulting from the blunt tips. The idea is briefly illustrated in Fig.1. We use a conventional glass axicon with a large base angle, which exhibits a rounded tip region much smaller compared to small base angle axicons. We then immerse this axicon in an index-matching liquid, with refractive index smaller than that of the axicon material. The effect of the liquid is to decrease the transverse spatial phase gradient and consequently to reduce the effective cone angle of the resulting Bessel-like beam. In other words, the presence of liquid behind a large-base-angle axicon decreases the angle of refraction of the rays forming the conical beam, with minimal aberrations caused by the round tip (since it covers a much smaller area for large base angles). This configuration has important advantages. First, since the index-matching liquids are available over a broad range $(1.3 \leq n \leq 2.3)$ with small increments $(\Delta n=0.002)$, the output cone angle can be adjusted by the choice of the index. Furthermore, by adjusting the temperature of the liquid, fine tuning can be achieved. Lastly, using a liquid with index of refraction very close to that of the axicon glass, very small cone angles can be achieved. A conventional axicon with such a small base angle would be both complicated to manufacture and prone to introduce strong aberrations. We confirm these principles both theoretically and experimentally. We also demonstrate the merit of the liquid immersion axicon in applications involving high-power femtosecond lasers by generating $\sim 2 \mathrm{~m}$ long plasma channels in air.

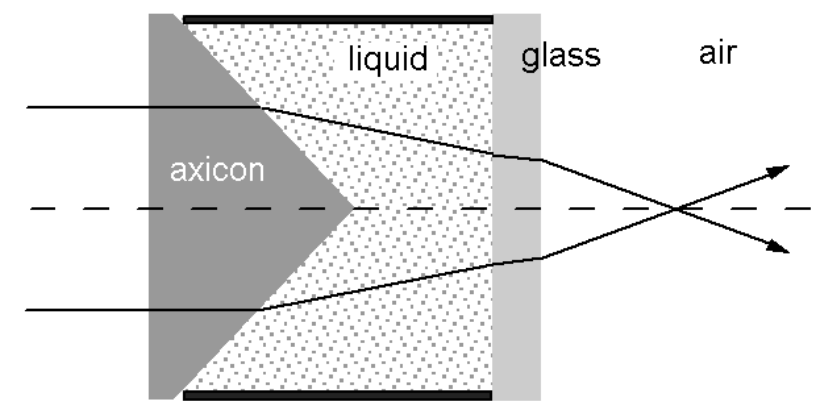

Fig. 1 The construction of the liquid immersion axicon. A large angle axicon is immersed in index-matching liquid, which is held in a ring shaped housing.

The idea of using fluids to construct tunable axicons was first demonstrated recently by Milne et al. [25], who used an inverse-molded polymer axicon filled with sucrose solution. By changing the sucrose concentration, the size of the core of the Bessel beam was adjusted. In our work, in addition to tunability, we put a special emphasis on the quality of the on-axis intensity distribution (i.e. elimination of the modulations) as it is critical to perform reliable and unbiased experiments to understand the dynamics of nonlinear propagation of high power Bessel beams $[15,16]$. Furthermore, we also show that fine tunability can be achieved via adjusting the liquid temperature.

Alternative schemes were previously suggested to generate tunable Bessel-like beams including tunable acoustic gradients [20], imaging with lenses [26, 27] and using the electrooptic effect in a uniaxial nonlinear crystal [28]. The aberrations resulting from the blunt tips can also be reduced by spatial filtering in the focal plane of the primary lens used for imaging [24]. 
Compared to these schemes the liquid-immersion axicon provides a more compact, higher throughput and better beam quality solution. Furthermore, schemes involving imaging pose difficulties in high power laser applications since the beam goes through a focus and can generate nonlinear interactions (such as plasma formation) before the Bessel zone.

\section{Liquid immersion axicon: theoretical and experimental results}

The propagation of beams after passing through an axicon is primarily determined by the half cone angle $\beta$ of the resulting $k$-vectors, as the transverse electric field profile is of the form $E(r) \propto J_{0}(k r \sin \beta)$, where $J_{0}$ is the zeroth-order Bessel function of the first kind, $k$ is the wavenumber and $r$ is the radial distance. The cone angle for the liquid immersion axicon can be calculated for a collimated input beam, using Snell's law:

$$
\beta=\arcsin \left[n_{l} \sin \left(\alpha_{l}-\alpha_{a}\right)\right]
$$

where $n_{l}$ and $n_{a}$ are indices of refraction for the liquid and axicon, $\alpha_{a}$ is the axicon base angle and $\alpha_{l}=\arcsin \left(n_{a} \sin \alpha_{a} / n_{l}\right)$ is the angle that the beam makes with the normal in liquid at the axicon-liquid interface. For small angles, this reduces to:

$$
\beta=n_{l}\left(\alpha_{l}-\alpha_{a}\right)
$$

The angle $\beta$ can be adjusted by changing $n_{l}$, either by choosing a different liquid or by adjusting the temperature. The change of index of refraction with the temperature is given by $n(T)=n_{0}+\Delta T \times d n / d T$, where $\Delta T$ is the change in the temperature and $d n / d T$ is the temperature coefficient for the index. For index-matching liquids $d n / d T$ is typically negative.

In order to demonstrate these ideas experimentally, we used an axicon of $25^{\circ}$ base angle (50 mm diameter, made of fused silica, by Doric lenses). Applying the recipe presented in [22], we confirmed using a small $(0.9 \mathrm{~mm}$ diameter $)$ input beam that the round area of the tip is negligible (much smaller than the beam size). We designed a ring-like housing to sandwich the liquid between the axicon and a glass window (see Fig. 1). Note that the use of a glass window with parallel faces has no effect on the output beam profile. We have used index matching liquid (Cargille Labs, series AA) with refractive index of 1.45 at D line $\left(589.3 \mathrm{~nm}\right.$ ) at $25{ }^{\circ} \mathrm{C}$. We used a He-Ne laser (at $633 \mathrm{~nm}$ wavelength) with beam diameter expanded to $3.74 \mathrm{~mm}$. After we passed the beam through the liquid-immersion axicon, we measured the transverse intensity profile at different propagation distances by using a CCD camera (UEye 2210M). Figure 2 (left) shows a typical measured transverse beam profile (square root taken for clarity). This profile exhibits the expected Bessel-like form with no visible aberrations. The radial variation of the intensity fits well with ideal Bessel function squared, as compared in Fig.2 (right). Using the transverse profiles, we also measured the evolution of the on axis intensity as a function of the propagation distance. 

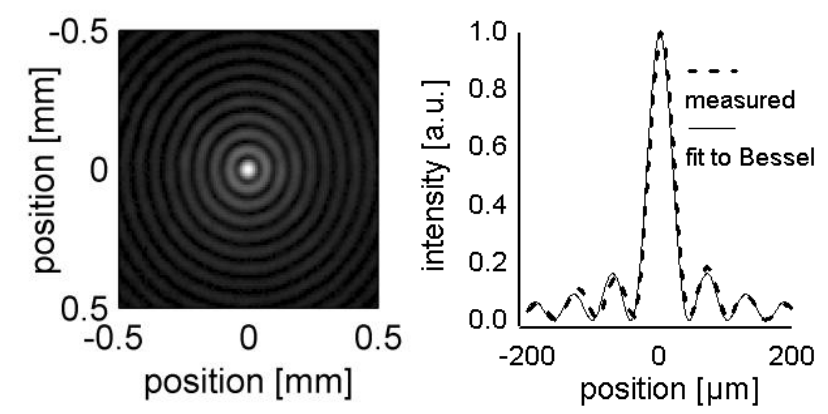

Fig. 2 Left: The measured beam profile after the liquid immersion axicon exhibits Bessel-like transverse intensity (square root taken for clarity of lower intensity rings). Right: Measured radial intensity profile (dashed line) fitted to Bessel function squared (solid line).

In order to compare the measured profile with the theoretical predictions, we calculated the light electric field behind the axicon. In the case of Gaussian input beam, the intensity distribution behind an ideal axicon can be calculated analytically using the stationary phase approximation [29, 30]:

$$
I(r, z)=\frac{4 P k r \sin \beta}{w_{0}} \frac{z}{z_{\max }} J_{0}^{2}(k r \sin \beta) \exp \left(-\frac{2 z^{2}}{z_{\max }^{2}}\right)
$$

where $P$ is the total power in the beam, $w_{0}$ is the beam spot size at waist, $z$ is the longitudinal position and $z_{\text {maz }}=w_{0} / \tan \beta$ is the position at which maximum axial intensity occurs. In order to take into account the effect of the blunt tip, we numerically solved the Fresnel-Kirschoff integral $[22,31]$ (which gives the same results of Eq.(3) for ideal axicon):

$$
E(r, z)=\frac{1}{i \lambda z} \exp \left(i k z+\frac{r^{2}}{2 z}\right) \int_{0}^{\infty} \exp \left(\frac{i k}{2 z} r_{0}^{2}\right) J_{0}\left(k r_{0} r_{1} / z\right) E\left(r_{0}\right) r_{0} d r_{0}
$$

where $\lambda$ is the wavelength. The input beam is taken to be a Gaussian with the measured diameter and with spatial phase introduced by the axicon: $\phi(r) \propto k r \tan \alpha_{a}\left(n_{l}-n_{a}\right)$ :

$$
E\left(r_{0}\right)=E_{0} \exp \left(-\frac{r^{2}}{w_{0}^{2}}-i k \frac{r^{2}}{2 R}\right) \exp \left(i \phi\left(r_{0}\right)\right)
$$

where $R$ is the beam radius of curvature. To evaluate $\phi(r)$, the index of refraction of the liquid at the laser wavelength is calculated using the measured indices at three different wavelengths (provided by the manufacturer) in the Hartmann dispersion formula [32]. The index of the axicon material is calculated using the Sellmeier's formula. These indices are then adjusted for the temperature of the element using the expression given above (for the liquid $d n / d T=-3.78 \times 10^{-4} /{ }^{\circ} \mathrm{C}$, for fused silica $\left.d n / d T=1.28 \times 10^{-5} /{ }^{\circ} \mathrm{C}\right)$. 

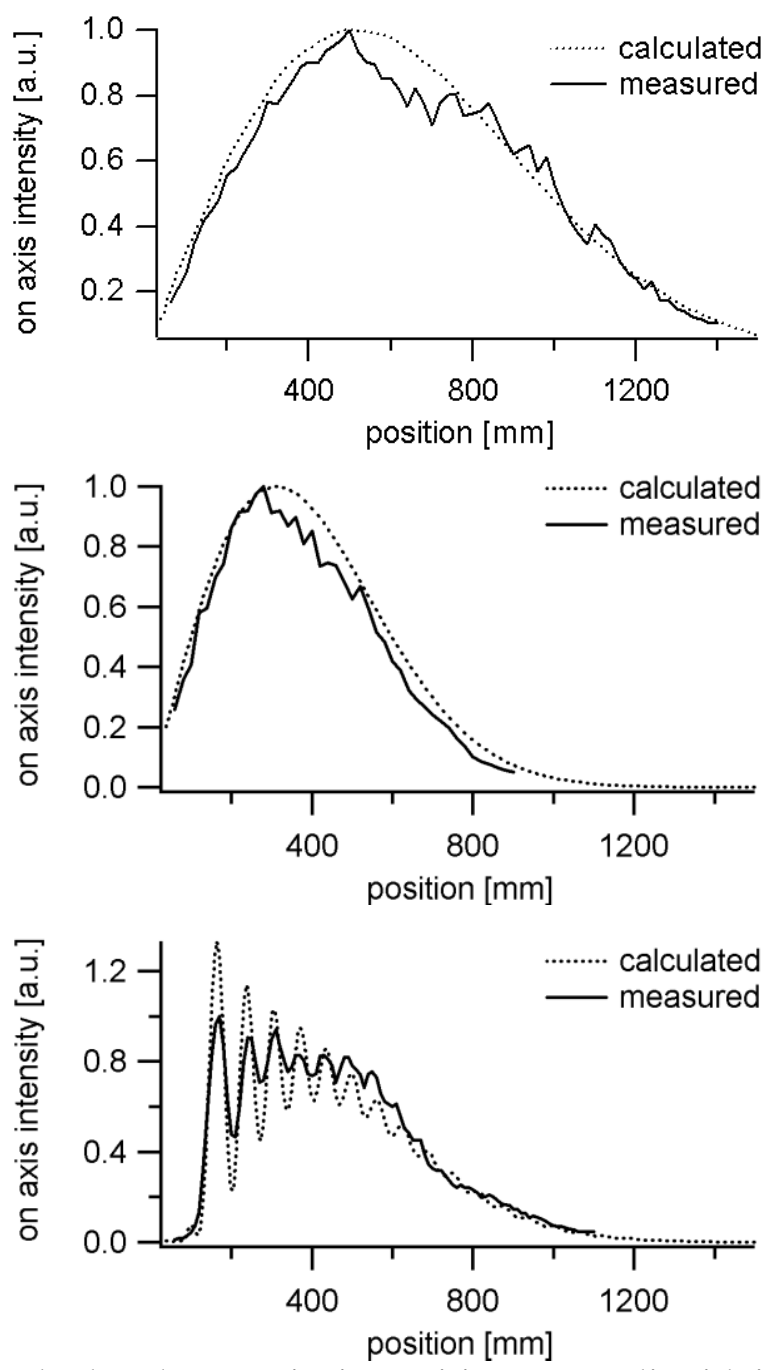

Fig. 3 Measured and calculated on axis intensities. Top: liquid immersion axicon at $20^{\circ} \mathrm{C}$, Center: liquid immersion axicon at $31^{\circ} \mathrm{C}$, Bottom: Conventional $0.5^{\circ}$ base angle axicon.

Figure 3 (top) shows the measured and calculated on-axis intensity profiles after focusing through the liquid-immersion axicon (at $20^{\circ} \mathrm{C}$ ). It can be seen that the choice of the liquid index very close to that of the axicon material allows the generation of a focal line longer than $1.3 \mathrm{~m}$. To generate this cone angle $\left(\beta=0.18^{\circ}\right)$ and focal depth with a regular axicon would require $\sim 0.4^{\circ}$ base angle (with fused silica). More importantly, one can note that there are no oscillations on the on-axis intensity as observed in the case of regular small angle axicons (see Fig.3, bottom, and [22]). To demonstrate the tunability through temperature adjustment, we increased the temperature of the liquid immersion axicon to $31{ }^{\circ} \mathrm{C}$, and repeated the measurements. Figure 3 (center) shows the measurements and calculations for this temperature. Evidently, the adjustment of merely $11{ }^{\circ} \mathrm{C}$ allowed us to increase the cone angle significantly $\left(\beta=0.28^{\circ}\right)$ and reduce the focal line to $\sim 0.8 \mathrm{~m}$. The advantages of the scheme we demonstrate become clearer through comparison with a regular axicon. We used $0.5^{\circ}$ base angle axicon (made of BK7 glass) and repeated the measurements (Figure 3, bottom). The calculations are performed taking into account the blunt-tip profile [22]. The on axis intensity in this case exhibits significant 
oscillations, resulting from the interference of the rays emerging from the lens-like tip and the axicon-like surroundings.

\section{Generation of plasma channels in air with the liquid-immersion axicon}

In order to demonstrate the feasibility of using the liquid immersion axicon in nonlinear optical applications involving high peak power lasers, we used a Ti:Sapphire laser system (800 $\mathrm{nm}$ center wavelength, $50 \mathrm{fs}$ pulse duration, $6 \mathrm{~mJ}$ pulse energy, $10 \mathrm{~mm}$ beam diameter). By focusing these pulses with the liquid immersion axicon, we generated a plasma channel in air through multiphoton and tunnel ionization [33] (Generation of plasma channels in air are of significant interest for several applications [17, 34, 35]). The presence of plasma is detected using two electrodes with high $(7 \mathrm{kV})$ potential difference, and measuring the current through the grounded electrode, resulting from the plasma screening (more details on the detection in [15]). Figure 4 shows the measured current at different transverse positions. The overall length of the plasma channel reaches $\sim 2 \mathrm{~m}$. The free electron density is free of oscillations observed in similar experiments performed with a regular axicon $[15,16]$. We observed that when the input pulse energy is further increased $(\mathrm{E}>6 \mathrm{~mJ})$, the beam starts to form small-scale filaments within the liquid. This limitation can be addressed either by minimizing the liquid thickness (using smaller axicon-glass separation in Fig.1) or by increasing the input beam diameter.

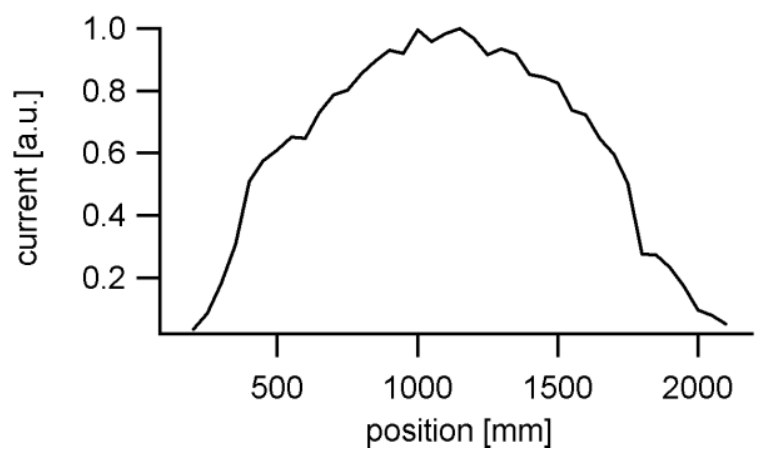

Fig. 4 Detection of plasma channel generated by focusing ultrashort pulses with the liquid immersion axicon. The measured induced current indicates presence of plasma over $\sim 2 \mathrm{~m}$.

\section{Conclusion}

In conclusion, we demonstrated a novel optical element, liquid immersion axicon, which can be used to generate Bessel-like beams in tunable manner and with minimal aberrations. We generated line foci longer than what could be achieved with regular axicons, and the resulting on-axis intensity was found to be free from modulations. We also used the liquid immersion axicon to focus high power ultrashort laser pulses and generated $\sim 2 \mathrm{~m}$ long plasma channels in air.

We gratefully acknowledge the funding of C. L. Arnold by the Deutsche Akademie der Naturforscher Leopoldina, Grant No. BMBF-LPD 9901/8-181.

\section{References}

1. J. Durnin, "Exact solutions for nondiffracting beams. I. the scalar theory," J. Opt. Soc. Am. A 4, 651-654 (1987). 
2. Z. Ding, H. Ren, Y. Zhao, J. S. Nelson, and Z. Chen, "High-resolution optical coherence tomography over a large depth range with an axicon lens," Opt. Lett. 27, 243-245 (2002).

3. P. Dufour, M. Piché, Y. De Koninck, and N. McCarthy, "Two-photon excitation fluorescence microscopy with a high depth of field using an axicon," Appl. Opt. 45, 9246-9252 (2006).

4. P. Polesana, D. Faccio, P. Di Trapani, A. Dubietis, A. Piskarkas, A. Couairon, and M. A. Porras, "High localization, focal depth and contrast by means of nonlinear Bessel Beams," Opt. Express 13, 6160-6167 (2005).

5. L. Paterson, E. Papagiakoumou, G. Milne, V. Garces-Chavez, S. A. Tatarkova, W. Sibbett, F. J. Gunn-Moore, P. E. Bryant, A. C. Riches, and K. Dholakia, "Light-induced cell separation in a tailored optical landscape," Appl. Phys. Lett. 87, 123901-123903 (2005).

6. T. Cizmár, V. Kollárová, X. Tsampoula, F. Gunn-Moore, W. Sibbett, Z. Bouchal, and K. Dholakia, "Generation of multiple Bessel beams for a biophotonics workstation," Opt. Express 16, 14024-14035 (2008).

7. V. Garces-Chavez, D. McGloin, H. Melville, W. Sibbett, and K. Dholakia, "Simultaneous micromanipulation in multiple planes using a self-reconstructing light beam," Nature 419, 145-147 (2002).

8. P. Polesana, A. Dubietis, M. A. Porras, E. Kucinskas, D. Faccio, A. Couairon, and P. Di Trapani, "Near-field dynamics of ultrashort pulsed Bessel beams in media with Kerr nonlinearity," Phys. Rev. E 73, 056612 (2006).

9. V. Pyragaite, K. Regelskis, V. Smilgevicius, and A. Stabinis, "Self-action of Bessel light beams in medium with large nonlinearity," Opt. Commun. 257, 139-145 (2006).

10. J. Arlt, K. Dholakia, L. Allen, and M. J. Padgett, "Efficiency of second-harmonic generation with Bessel beams," Phys. Rev. A 60, 2438 (1999).

11. P. Polesana, M. Franco, A. Couairon, D. Faccio, and P. Di Trapani, "Filamentation in Kerr media from pulsed bessel beams," Phys. Rev. A 77, 043814 (2008).

12. A. Dubietis, P. Polesana, G. Valiulis, A. Stabinis, P. Di Trapani, and A. Piskarskas, "Axial emission and spectral broadening in self-focusing of femtosecond Bessel beams," Opt. Express 15, 4168-4175 (2007).

13. P. Polesana, A. Couairon, D. Faccio, A. Parola, M. A. Porras, A. Dubietis, A. Piskarskas, and P. Di Trapani, "Observation of Conical Waves in Focusing, Dispersive, and Dissipative Kerr Media," Phys. Rev. Lett. 99, 223902-223904 (2007).

14. C. G. Durfee and H. M. Milchberg, "Light pipe for high intensity laser pulses," Phys. Rev. Lett. 71, 2409-2412 (1993).

15. S. Akturk, B. Zhou, M. Franco, A. Couairon, and A. Mysyrowicz, "Generation of long plasma channels in air by focusing ultrashort laser pulses with an axicon," Opt. Commun. 282, 129-134 (2008).

16. P. Polynkin, M. Kolesik, A. Roberts, D. Faccio, P. Di Trapani, and J. Moloney, "Generation of extended plasma channels in air using femtosecond Bessel beams," Opt. Express 16, 15733-15740 (2008).

17. G. Roy, M. Blanchard, and R. Tremblay, "High-pressure amplified stimulated emission effect in a N2 laser produced plasma with axicon lenses," Opt. Commun. 33, 65-68 (1980).

18. J. Durnin, J. J. Miceli, and J. H. Eberly, "Diffraction-free beams," Phys. Rev. Lett. 58, 1499-1501 (1987). 
19. L. Niggl, T. Lanzl, and M. Maier, "Properties of Bessel beams generated by periodic gratings of circular symmetry," J. Opt. Soc. Am. A 14, 27-33 (1997).

20. E. McLeod, A. B. Hopkins, and C. B. Arnold, "Multiscale Bessel beams generated by a tunable acoustic gradient index of refraction lens," Opt. Lett. 31, 3155-3157 (2006).

21. J. H. McLeod, "Axicons and their uses," J. Opt. Soc. Am. 50, 166-169 (1960).

22. S. Akturk, B. Zhou, B. Pasquiou, M. Franco, and A. Mysyrowicz, "Intensity distribution around the focal regions of real axicons," Opt. Commun. 281, 4240-4244 (2008).

23. B. Depret, P. Verkerk, and D. Hennequin, "Characterization and modelling of the hollow beam produced by a real conical lens," Opt. Commun. 211, 31-38 (2002).

24. O. Brzobohatý, T. Cizmár, and P. Zemánek, "High quality quasi-Bessel beam generated by round-tip axicon," Opt. Express 16, 12688-12700 (2008).

25. G. Milne, G. D. M. Jeffries, and D. T. Chiu, "Tunable generation of Bessel beams with a fluidic axicon," Appl. Phys. Lett. 92, 261101-261103 (2008).

26. S. Klewitz, F. Brinkmann, S. Herminghaus, and P. Leiderer, "Bessel-beam-pumped tunable distributed-feedback laser," Appl. Opt. 34, 7670-7673 (1995).

27. V. Vaičaitis and Š. Paulikas, "Formation of Bessel beams with continuously variable cone angle," Opt. Quantum Electron. 35, 1065-1071 (2003).

28. N. Kazak, M. Krening, A. Mashchenko, and P. Ropot, "A controlled conical lens for the formation of Bessel light beams," Opt. Spectrosc. 103, 800-803 (2007).

29. G. Roy and R. Tremblay, "Influence of the divergence of a laser beam on the axial intensity distribution of an axicon," Opt. Commun. 34, 1-3 (1980).

30. J. Arlt and K. Dholakia, "Generation of high-order Bessel beams by use of an axicon," Opt. Commun. 177, 297-301 (2000).

31. M. Born and E. Wolf, Principles of Optics: Electromagnetic Theory of Propagation, Interference and Diffraction of Light (Cambridge Univ Pr, 1999).

32. E. Akatsu, "Physical meaning of coefficients for glass compositions determined by Hartmann's dispersion formula," Appl. Opt. 21, 1896-1897 (1982).

33. A. Couairon and A. Mysyrowicz, "Femtosecond filamentation in transparent media," Phys. Rep. 441, 47-189 (2007).

34. S. Tzortzakis, B. Prade, M. Franco, A. Mysyrowicz, S. Hüller, and P. Mora, "Femtosecond laser-guided electric discharge in air," Phys. Rev. E 64, 057401 (2001).

35. M. Rodriguez, R. Sauerbrey, H. Wille, L. Wöste, T. Fujii, Y.-B. André, A. Mysyrowicz, L. Klingbeil, K. Rethmeier, W. Kalkner, J. Kasparian, E. Salmon, J. Yu, and J.-P. Wolf, "Triggering and guiding megavolt discharges by use of laser-induced ionized filaments " Opt. Lett. 27, 772-774 (2002). 